\title{
Chernoff-Based Multi-class Pairwise Linear Dimensionality Reduction
}

\author{
Luis Rueda ${ }^{1, \star}$, Claudio Henríquez ${ }^{2}$, and B. John Oommen ${ }^{3, \star \star}$ \\ ${ }^{1}$ School of Computer Science, University of Windsor, 401 Sunset Ave., Windsor, ON, \\ N9P 3P4, Canada \\ lrueda@uwindsor.ca \\ ${ }^{2}$ Department of Computer Science, University of Concepción, Edmundo Larenas 215, \\ Concepción, 4070409, Chile \\ chenriquezv@udec.cl \\ ${ }^{3}$ School of Computer Science, Carleton University, 1125 Colonel By Dr., Ottawa, \\ ON, K1S 5B6, Canada \\ oommen@scs . carleton. ca
}

\begin{abstract}
Linear dimensionality reduction techniques have been studied very well for the two-class problem, while the corresponding issues encountered when dealing with multiple classes are far from trivial. In this paper, we show that dealing with multiple classes, it is not expedient to treat it as a multi-class problem, but it is better to treat it as an ensemble of Chernoff-based two-class reductions onto different subspaces. The solution is achieved by resorting to either Voting, Weighting, or a Decision Tree combination scheme. The ensemble methods were tested on benchmark datasets demonstrating that the proposed method is not only efficient, but also yields an accuracy comparable to that obtained by the optimal Bayes classifier.
\end{abstract}

Keywords: Linear Dimensionality Reduction, Fisher's Discriminant Analysis, Heteroscedastic Discriminant Analysis, Chernoff Distance.

\section{Introduction}

Linear dimensionality reduction (LDR) is an important field on pattern recognition for which the aim is to extract and retain only the efficient features that yield superior classification in the reduced subspace, providing a reliable classification with the input data. The most traditional LDR scheme is the Fisher's discriminant approach (FDA), and its many extensions 4. An improvement to FDA that decomposes classes into subclasses has been proposed in [5]. Also, a scheme to find an optimal kernel over a convex set of kernels has been recently proposed for the kernelized FDA [3. Optimal pairwise linear classifiers can also be seen as an alternate way of linearly reducing the dimensionality of the space

\footnotetext{
* Member of the IEEE.

** Chancellor's Professor. Fellow of the IEEE and IAPR. This author is also an Adjunct Professor with the University of Agder in Grimstad, Norway.
} 
in which the classification is done [9], and a related approach to selecting the best hyperplane classifier (BHC) [7, while in [6], a computationally intensive method for LDR was proposed. Another criterion used for dimensionality reduction is the subclass discriminant analysis [11], which aims to optimally divide the classes into subclasses, and then perform a reduction followed by classification.

Two approaches have been proposed in order to generalize homoscedastic-like approaches. One of these schemes is the heteroscedastic discriminant analysis (HDA) approach proposed in 4, which considers the concept of directed distance matrices, and the so-called Chernoff-based discriminant analysis (CDA) scheme [8], which maximizes the Chernoff distance of the lower-dimensional classes. Both HDA and CDA have been proposed for multi-class problems, providing a solution in terms of a single transformation matrix that projects from the higher-dimensional space to a lower-dimensional space.

Among schemes that configure multiple transformations, we have the voting rule as proposed in [10], the one-against-all, one-against-one, and all-at-once strategies, and the use of decision trees [1 for generalizing two-class Support Vector Machines (SVMs) for multi-class problems so as to avoid unclassifiable regions. In this paper, we propose a multi-class LDR scheme as an ensemble of two-class LDR schemes - rather than resorting to a single multi-class LDR schemes. We perform a systematic study of how the results of the individual twoclass classifiers can be fused, i.e., by either a Voting, Weighting, or a Decision Tree strategy. The results on a testing using benchmark datasets show that the one which involves a Chernoff-based criterion [8] is the most suitable one.

\section{Two-Class LDR Schemes}

For two classes, we assume that they are given by their a priori probabilities, $p_{1}$ and $p_{2}$, and two $n$-dimensional normally distributed random vectors, $\mathbf{x}_{1} \sim$ $N\left(\mathbf{m}_{1} ; \mathbf{S}_{1}\right)$ and $\mathbf{x}_{2} \sim N\left(\mathbf{m}_{2} ; \mathbf{S}_{2}\right)$. The problem is to find a $d \times n$ matrix $\mathbf{A}$ in such a way that the transformed data, given by the linear transformation $\mathbf{y}_{i}=\mathbf{A} \mathbf{x}_{i}$, become as separable as possible. Let $\mathbf{S}_{W}=p_{1} \mathbf{S}_{1}+p_{2} \mathbf{S}_{2}$ and $\mathbf{S}_{E}=$ $\left(\mathbf{m}_{1}-\mathbf{m}_{2}\right)\left(\mathbf{m}_{1}-\mathbf{m}_{2}\right)^{t}$ be the within-class and between-class scatter matrices respectively. The FDA criterion consists of finding $\mathbf{A}$ that maximizes $J_{F}(\mathbf{A})=$ $\operatorname{tr}\left\{\left(\mathbf{A} \mathbf{S}_{W} \mathbf{A}^{t}\right)^{-1}\left(\mathbf{A} \mathbf{S}_{E} \mathbf{A}^{t}\right)\right\}$, , by taking the $d$ eigenvectors (whose eigenvalues are the largest ones) $\mathbf{S}_{W}^{-1} \mathbf{S}_{E}$. Since $\mathbf{S}_{E}$ is of rank unity, $\mathbf{S}_{W}^{-1} \mathbf{S}_{E}$ is also of rank unity. Thus, the eigenvalue decomposition of $\mathbf{S}_{W}^{-1} \mathbf{S}_{E}$ leads to only a single nonzero eigenvalue, and hence the FDA can only reduce to dimension $d=1$.

The HDA criterion consists of finding the matrix $\mathbf{A}$ that maximizes: $J_{L D_{2}}(\mathbf{A})$ $=\operatorname{tr}\left\{\left(\mathbf{A} \mathbf{S}_{W} \mathbf{A}^{t}\right)^{-1}\left[\mathbf{A} \mathbf{S}_{E} \mathbf{A}^{t}-\mathbf{A} \mathbf{S}_{W}^{\frac{1}{2}} \frac{p_{1} \log \left(\mathbf{S}_{W}^{-\frac{1}{2}} \mathbf{S}_{1} \mathbf{S}_{W}^{-\frac{1}{2}}\right)+p_{2} \log \left(\mathbf{S}_{W}^{-\frac{1}{2}} \mathbf{S}_{2} \mathbf{S}_{W}^{-\frac{1}{2}}\right)}{p_{1} p_{2}} \mathbf{S}_{W}^{\frac{1}{2}} \mathbf{A}^{t}\right]\right\}$, where the logarithm of a matrix $\mathbf{M}$ is given by $\log (\mathbf{M})=\boldsymbol{\Phi} \log (\boldsymbol{\Lambda}) \boldsymbol{\Phi}^{-1}$, with $\boldsymbol{\Phi}$ and $\boldsymbol{\Lambda}$ representing the eigenvectors and eigenvalues of $\mathbf{M}$ respectively. The solution is given by the $d$ eigenvectors (whose eigenvalues are the largest ones) of the matrix $\mathbf{S}_{W}^{-1}\left[\mathbf{S}_{E}-\mathbf{S}_{W}^{\frac{1}{2}} \frac{p_{1} \log \left(\mathbf{S}_{W}^{-\frac{1}{2}} \mathbf{S}_{1} \mathbf{S}_{W}^{-\frac{1}{2}}\right)+p_{2} \log \left(\mathbf{S}_{W}^{-\frac{1}{2}} \mathbf{S}_{2} \mathbf{S}_{W}^{-\frac{1}{2}}\right)}{p_{1} p_{2}} \mathbf{S}_{W}^{\frac{1}{2}}\right]$. 
It has been noted in 8] that HDA does not guarantee that the Chernoff distance in the transformed space is maximized, and this is what is proposed in [8]. The aim of CDA is to find the matrix $\mathbf{A}$ that maximizes $J_{C_{12}}^{*}(\mathbf{A})=$ $\operatorname{tr}\left\{p_{1} p_{2} \mathbf{A} \mathbf{S}_{E} \mathbf{A}^{t}\left(\mathbf{A} \mathbf{S}_{W} \mathbf{A}^{t}\right)^{-1}+\log \left(\mathbf{A} \mathbf{S}_{W} \mathbf{A}^{t}\right)-p_{1} \log \left(\mathbf{A} \mathbf{S}_{1} \mathbf{A}^{t}\right)-p_{2} \log \left(\mathbf{A} \mathbf{S}_{2} \mathbf{A}^{t}\right)\right\}$ where $\mathbf{S}_{W}=p_{1} \mathbf{S}_{1}+p_{2} \mathbf{S}_{2} . J_{C_{12}}^{*}$ is maximized via a gradient-based method. First, the gradient matrix is found by deriving the corresponding gradient operator, $\nabla J_{C_{12}}^{*}(\mathbf{A})$. Thereafter, the algorithm finds the maximum value of the learning rate at step $k, \eta_{k}$, by maximizing the objective function in the direction of the gradient. The new gradient matrix at step $k$ is obtained as $\mathbf{A}^{(k)}+\eta_{k} \nabla J_{C_{12}}^{*}\left(\mathbf{A}^{(k)}\right)$, and the process is repeated until the change between the objective functions at the current and previous steps is below a user-defined threshold.

\section{All-at-Once Multi-class Schemes}

The multi-class problem that we consider assumes $c$ classes, $\omega_{1}, \ldots, \omega_{c}$, given in terms of their a priori probabilities $p_{1}, \ldots, p_{c}$, and $c n$-dimensional normally distributed random vectors, $\mathbf{x}_{1} \sim N\left(\mathbf{m}_{1} ; \mathbf{S}_{1}\right), \ldots, \mathbf{x}_{c} \sim N\left(\mathbf{m}_{c} ; \mathbf{S}_{c}\right)$. In the allat-once scheme, the problem consists of finding a $d \times n$ transformation matrix $\mathbf{A}$ in such a way that the transformed data, given by the linear transformation $\mathbf{y}=\mathbf{A x}$, become as separable as possible.

For the FDA criterion, the following definitions are used: $\mathbf{S}_{E}=\sum_{i=1}^{c} p_{i}\left(\mathbf{m}_{i}-\right.$ $\mathbf{m})\left(\mathbf{m}_{i}-\mathbf{m}\right)^{t}$, where $\mathbf{m}=\sum_{i=1}^{c} p_{i} \mathbf{m}_{i}$, and $\mathbf{S}_{W}=\sum_{i=1}^{c} p_{i} \mathbf{S}_{i}$. The aim is to find a $d \times n$ transformation matrix $\mathbf{A}$ that maximizes the criterion function $J_{F}(\mathbf{A})=\operatorname{tr}\left\{\left(\mathbf{A} \mathbf{S}_{W} \mathbf{A}^{t}\right)^{-1}\left(\mathbf{A} \mathbf{S}_{E} \mathbf{A}^{t}\right)\right\}$, and which is obtained by finding the $d$ eigenvectors (whose eigenvalues are the largest) of the matrix $\mathbf{S}_{W}^{-1} \mathbf{S}_{E}$. Since $\mathbf{S}_{E}$ is of rank $c-1$, only this number of eigenvalues are nonzero, and so FDA can, at most, reduce the dimension to a value $c-1$.

The multi-class HDA criterion aims to find the $d \times n$ transformation matrix A that maximizes [4]:

$$
\begin{aligned}
& J_{L D}(\mathbf{A})=\sum_{i=1}^{c-1} \sum_{j=i+1}^{c} p_{i} p_{j} \operatorname{tr}\left\{\left(\mathbf{A} \mathbf{S}_{W} \mathbf{A}^{t}\right)^{-1} \mathbf{A} \mathbf{S}_{W}^{\frac{1}{2}}\right. \\
& \quad\left[\left(\mathbf{S}_{W}^{-\frac{1}{2}} \mathbf{S}_{i j} \mathbf{S}_{W}^{-\frac{1}{2}}\right)^{-\frac{1}{2}} \mathbf{S}_{W}^{-\frac{1}{2}} \mathbf{S}_{E_{i j}} \mathbf{S}_{W}^{-\frac{1}{2}}\left(\mathbf{S}_{W}^{-\frac{1}{2}} \mathbf{S}_{i j} \mathbf{S}_{W}^{-\frac{1}{2}}\right)^{-\frac{1}{2}}+\frac{1}{\pi_{i} \pi_{j}}\left(\log \left(\mathbf{S}_{W}^{-\frac{1}{2}} \mathbf{S}_{i j} \mathbf{S}_{W}^{-\frac{1}{2}}\right)\right.\right. \\
& \left.\left.\left.\quad-\pi_{i} \log \left(\mathbf{S}_{W}^{-\frac{1}{2}} \mathbf{S}_{i} \mathbf{S}_{W}^{-\frac{1}{2}}\right)-\pi_{j} \log \left(\mathbf{S}_{W}^{-\frac{1}{2}} \mathbf{S}_{j} \mathbf{S}_{W}^{-\frac{1}{2}}\right)\right)\right] \mathbf{S}_{W}^{\frac{1}{2}} \mathbf{A}^{t}\right\}
\end{aligned}
$$

where $\mathbf{S}_{E_{i j}}=\left(\mathbf{m}_{i}-\mathbf{m}_{j}\right)\left(\mathbf{m}_{i}-\mathbf{m}_{j}\right)^{t}, \pi_{i}=\frac{p_{i}}{p_{i}+p_{j}}, \pi_{j}=\frac{p_{j}}{p_{i}+p_{j}}$, and $\mathbf{S}_{i j}=\pi_{i} \mathbf{S}_{i}+$ $\pi_{j} \mathbf{S}_{j}$. The multi-class HDA criterion is maximized by finding the matrix $\mathbf{A}$ composed of the $d$ eigenvectors (whose eigenvalues are the largest ones) of the matrix $\mathbf{S}_{L D}=\sum_{i=1}^{c-1} \sum_{j=i+1}^{c} p_{i} p_{j} \mathbf{S}_{W}^{-1} \mathbf{S}_{W}^{\frac{1}{2}}\left[\left(\mathbf{S}_{W}^{-\frac{1}{2}} \mathbf{S}_{i j} \mathbf{S}_{W}^{-\frac{1}{2}}\right)^{-\frac{1}{2}} \mathbf{S}_{W}^{-\frac{1}{2}} \mathbf{S}_{E_{i j}} \mathbf{S}_{W}^{-\frac{1}{2}}\left(\mathbf{S}_{W}^{-\frac{1}{2}} \mathbf{S}_{i j} \mathbf{S}_{W}^{-\frac{1}{2}}\right)^{-\frac{1}{2}}\right.$ $\left.+\frac{1}{\pi_{i} \pi_{j}}\left(\log \left(\mathbf{S}_{W}^{-\frac{1}{2}} \mathbf{S}_{i j} \mathbf{S}_{W}^{-\frac{1}{2}}\right)-\pi_{i} \log \left(\mathbf{S}_{W}^{-\frac{1}{2}} \mathbf{S}_{i} \mathbf{S}_{W}^{-\frac{1}{2}}\right)-\pi_{j} \log \left(\mathbf{S}_{W}^{-\frac{1}{2}} \mathbf{S}_{j} \mathbf{S}_{W}^{-\frac{1}{2}}\right)\right)\right] \mathbf{S}_{W}^{\frac{1}{2}}$.

The multi-class CDA criterion is also an extension of the two-class case, and is obtained by maximizing the weighted sum of the pairwise Chernoff distances 
between classes $\omega_{i}$ and $\omega_{j}$, for all $i=1, \ldots, c-1, j=i, \ldots, c$. The weights used for the pairwise class criterion are given by the normalized joint prior probabilities between classes $\omega_{i}$ and $\omega_{j}$, namely, $\pi_{i} \pi_{j}$. The criterion consists of finding the optimal $d \times n$ transformation $\mathbf{A}$, in such a way that the following function is maximized:

$$
J_{C}^{*}(\mathbf{A})=\sum_{i=1}^{c-1} \sum_{j=i+1}^{c} J_{C_{i j}}^{*}(\mathbf{A}),
$$

where $J_{C_{i j}}^{*}(\mathbf{A})=\operatorname{tr}\left\{\pi_{i} \pi_{j}\left(\mathbf{A} \mathbf{S}_{W_{i j}} \mathbf{A}^{t}\right)^{-1} \mathbf{A} \mathbf{S}_{E_{i j}} \mathbf{A}^{t}+\log \left(\mathbf{A} \mathbf{S}_{W_{i j}} \mathbf{A}^{t}\right)-\pi_{i} \log \left(\mathbf{A} \mathbf{S}_{i} \mathbf{A}^{t}\right)\right.$ $\left.-\pi_{j} \log \left(\mathbf{A} \mathbf{S}_{j} \mathbf{A}^{t}\right)\right\}$. The gradient matrix is given by the first-order necessary condition in terms of $\nabla J_{C}^{*}(\mathbf{A})$. Then, as in the two-class case, to find the matrix $\mathbf{A}$ that maximizes $J_{C}^{*}(\mathbf{A})$, a gradient-based algorithm was proposed in [8].

\section{Pairwise Multi-class Schemes}

In our scheme, we use the linear reduction to increase the separability of the classes, mapping the objects in the reduced space so as to apply a classifier in that space, which ideally is more suitable for classification than the original space. The two-class above-described LDR techniques, namely FDA, HDA and CDA, are coupled with a back-end classifier, to develop the new multi-class classifiers.

When dealing with more than two classes, the search for the best transformed space which uses LDR methods leads to various problems: the increase in the inter-class overlap, the decrease in the between-class separability, and the existence of class covariances which are unequal. The first two handicaps can be observed in Figure 1 In (a), the classes are linearly separable in the original space and substantially overlap in the transformed space. In (b), although the classes remain to be linearly separable in the transformed space, they became closer to each other than in the original space. In (c), the classes are much more separable in their respective pairwise projection hyperplanes. Thus, we need a distinct new way for treating the multi-class linear reduction problem, and hence, we propose three ways by which we can use two-class LDR methods for the multi-class case, namely those that include the Voting, Weighting, and Decision Tree strategies respectively.

Simple Voting: It consists of a training and testing phase. We first train all possible two-class classifiers using the available training data, and thus obtain $\left(\begin{array}{l}c \\ 2\end{array}\right)$ possible LDR classifiers. On encountering an unknown sample, $\mathbf{x}$, it is tested against all the $\left(\begin{array}{l}c \\ 2\end{array}\right)$ classifiers, and every class is given a vote of unity whenever it "wins" a two-class competition. Ultimately, $\mathbf{x}$ will be labelled to the class with the highest number of votes. In case of ties, the assignment is to the class with the higher a priori probability, or if ties are not broken, to a random class.

Weighted Voting: We first train all the possible two-class classifiers using the available training data. On encountering an unknown sample, $\mathbf{x}$, it is tested against all the $\left(\begin{array}{l}c \\ 2\end{array}\right)$ classifiers. For any two-class competition in which the classifier 


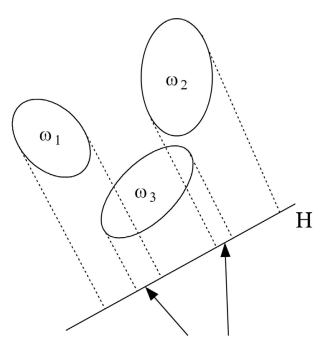

(a) Overlapping

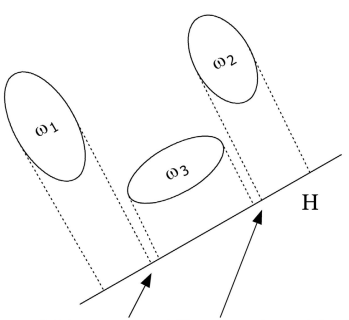

(b) Less separability than the original space

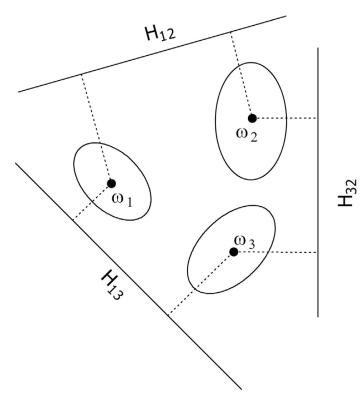

(c) Pairwise projection

Fig. 1. The effect on the overlapping between two classes and their separability by mapping them onto different lower-dimensional subspaces

involves classes $\omega_{i}$ and $\omega_{j}$ (represented in a subspace by hyperplane $H_{i j}$ ), the confidence of $\mathbf{x}$ belonging to these classes, say $V_{i}(\mathbf{x})$ and $V_{j}(\mathbf{x})$ respectively, is increased by $f_{i}(\mathbf{x})$ and $f_{j}(\mathbf{x})$ respectively, where: $f_{i}(\mathbf{x})=\frac{P_{H_{i j}}\left(\omega_{i} \mid \mathbf{x}\right)}{P_{H_{i j}}\left(\omega_{i} \mid \mathbf{x}\right)+P_{H_{i j}}\left(\omega_{j} \mid \mathbf{x}\right)}$ and $f_{j}(\mathbf{x})=\frac{P_{H_{i j}}\left(\omega_{j} \mid \mathbf{x}\right)}{P_{H_{i j}}\left(\omega_{i} \mid \mathbf{x}\right)+P_{H_{i j}}\left(\omega_{j} \mid \mathbf{x}\right)}$, with $P_{H_{i j}}\left(\omega_{i} \mid \mathbf{x}\right)$ and $P_{H_{i j}}\left(\omega_{j} \mid \mathbf{x}\right)$ being the two-class posterior probabilities of assigning $\mathbf{x}$ to $\omega_{i}$ and $\omega_{j}$ respectively.

Decision Tree: Following the decision trees proposed in [1, at every node, we classify $\mathbf{x}$ based on a local discriminant function, $D_{i j}$, which determines whether $\mathbf{x}$ should be assigned to class $\omega_{j}$ or class $\omega_{j}$, where $D_{i j}(\mathbf{x})$ is the result of a two-class $\left(\omega_{i}\right.$ vs. $\left.\omega_{j}\right)$ LDR and a classifier. By invoking a sequence of twoclass classifiers, we eliminate the classes to which $\mathbf{x}$ will not be assigned, and ultimately reach a leaf node where the final classification of $\mathbf{x}$ can be achieved. An advantage of using this scheme is that $O(c)$ decisions are made in order to classify a single sample, as opposed to the $\frac{c(c-1)}{2}=O\left(c^{2}\right)$ decisions needed in the voting and weighted schemes. However, even though the ambiguous region problem is resolved, the order of the decision making nodes in the tree affects the classification performance in most of the cases.

\section{Experimental Results}

The experiments were performed on six datasets taken from the UCI ML Repository [2]: Iris Plants, Pen-Based Recognition of Handwritten Digits, Thyroid Disease, Wine, Glass Identification, Vowel Recognition. Using these datasets, we performed a 10-fold cross-validation. Some PCA preprocessing was performed to Glass in order to avoid ill-conditioned covariance matrices, reducing the number of dimensions from nine to eight. We also removed class ' 6 ' because it contained less than 10 elements, affecting the 10-fold cross-validation.

The comparison was made on the basis of the three LDR techniques explained above, namely the FDA, HDA and CDA methods. Also, each of these methods was complemented with linear and quadratic classifiers in the transformed 
Table 1. Accuracies obtained with three LDR methods coupled with linear and quadratic classifiers, on datasets from the UCI ML repository

\begin{tabular}{|c|c|c|c|c|c|c|c|c|c|c|c|c|c|}
\hline Dataset & $n$ & $\mathrm{FDA}+\mathrm{L}$ & $d$ & $\mathrm{HDA}+\mathrm{L}$ & $d$ & $\mathrm{CDA}+\mathrm{L}$ & $d$ & $\mathrm{FDA}+\mathrm{Q}$ & $d$ & $\mathrm{HDA}+\mathrm{Q}$ & $d$ & $\mathrm{CDA}+\mathrm{Q}$ & $d$ \\
\hline \multicolumn{14}{|c|}{ All-at-once: } \\
\hline Iris & 4 & $0.9800^{*}$ & 1 & $0.9800^{*}$ & 1 & $0.9800^{*}$ & 1 & 0.9733 & 1 & $0.9800^{*}$ & 1 & $0.9800^{*}$ & 1 \\
\hline Pendigits & 16 & $0.8760^{*}$ & 9 & 0.8709 & 15 & 0.8699 & 15 & 0.9507 & 9 & 0.9768 & 15 & $0.9777^{*}$ & 14 \\
\hline Thyroid & 5 & $0.9065^{*}$ & 1 & $0.9065^{*}$ & 4 & $0.9065^{*}$ & 1 & $0.9671^{*}$ & 1 & 0.9578 & 1 & 0.9626 & 4 \\
\hline Wine & 13 & 0.9778 & 2 & $0.9889^{*}$ & 5 & 0.9836 & 2 & 0.9889 & 2 & $0.9945^{*}$ & 2 & $0.9945^{*}$ & 2 \\
\hline Glass & 8 & $0.6613^{*}$ & 4 & 0.6032 & 6 & 0.5894 & 6 & $0.5667^{*}$ & 2 & 0.5532 & 4 & 0.5504 & 4 \\
\hline Vowel & 10 & 0.5344 & 6 & $0.5556^{*}$ & 2 & $0.5556^{*}$ & 2 & 0.6212 & 9 & 0.6778 & 6 & $0.6960^{*}$ & 6 \\
\hline \multicolumn{14}{|c|}{ Simple Voting: } \\
\hline Iris & 4 & $0.9800^{*}$ & 1 & $0.9800^{*}$ & 4 & $0.9800^{*}$ & 1 & 0.9667 & 1 & $0.9800^{*}$ & 2 & $0.9800^{*}$ & 3 \\
\hline Pendigits & 16 & 0.9652 & 1 & 0.9655 & 16 & $0.9656^{*}$ & 2 & 0.9675 & 1 & 0.9813 & 15 & $0.9821 *$ & 15 \\
\hline Thyroid & 5 & 0.9160 & 1 & $0.9483^{*}$ & 4 & $0.9483^{*}$ & 4 & $0.9626^{*}$ & 1 & 0.9578 & 4 & 0.9578 & 5 \\
\hline Wine & 13 & 0.9830 & 1 & 0.9886 & 12 & 0.9889* & 12 & 0.9889 & 1 & 0.9889 & 12 & $0.9944^{*}$ & 7 \\
\hline Glass & 8 & 0.6334 & 1 & 0.6354 & 5 & $0.6586^{*}$ & 6 & $0.6080^{*}$ & 1 & 0.6051 & 8 & 0.6000 & 7 \\
\hline Vowel & 10 & 0.5980 & 1 & $0.6111^{*}$ & 4 & 0.6051 & 9 & 0.6030 & 1 & 0.6990 & 4 & $0.7131^{*}$ & 4 \\
\hline \multicolumn{14}{|c|}{ Weighted Voting: } \\
\hline Iris & 4 & $0.9800^{*}$ & 1 & $0.9800^{*}$ & 4 & $0.9800^{*}$ & 1 & 0.9667 & 1 & $0.9800^{*}$ & 2 & $0.9800^{*}$ & 3 \\
\hline Pendigits & 16 & $0.9681^{*}$ & 1 & 0.9680 & 16 & $0.9681^{*}$ & 1 & 0.9692 & 1 & 0.9813 & 15 & $0.9821^{*}$ & 15 \\
\hline Thyroid & 5 & 0.9160 & 1 & $0.9435^{*}$ & 4 & $0.9435 *$ & 4 & $0.9626^{*}$ & 1 & 0.9578 & 4 & 0.9532 & 1 \\
\hline Wine & 13 & 0.9830 & 1 & 0.9886 & 12 & $0.9889^{*}$ & 12 & 0.9889 & 1 & 0.9889 & 12 & $0.9944^{*}$ & 7 \\
\hline Glass & 8 & 0.6171 & 1 & 0.6213 & 5 & $0.6249^{*}$ & 6 & $0.6032 *$ & 1 & 0.5809 & 8 & 0.5864 & 7 \\
\hline Vowel & 10 & 0.6010 & 1 & $0.6182^{*}$ & 5 & 0.6081 & 1 & 0.6040 & 1 & 0.7010 & 4 & $0.7172^{*}$ & 4 \\
\hline \multicolumn{14}{|c|}{ Decision Tree: } \\
\hline Iris & 4 & $0.9800^{*}$ & 1 & $0.9800^{*}$ & 4 & $0.9800^{*}$ & 1 & 0.9667 & 1 & $0.9800^{*}$ & 2 & $0.9800^{*}$ & 3 \\
\hline Pendigits & 16 & 0.9624 & 1 & 0.9625 & 16 & $0.9626^{*}$ & 2 & 0.9658 & 1 & 0.9813 & 15 & $0.9821^{*}$ & 15 \\
\hline Thyroid & 5 & 0.9160 & 1 & $0.9483^{*}$ & 4 & $0.9483^{*}$ & 4 & $0.9626^{*}$ & 1 & 0.9578 & 4 & 0.9578 & 5 \\
\hline Wine & 13 & 0.9830 & 1 & 0.9886 & 12 & $0.9889^{*}$ & 12 & 0.9889 & 1 & 0.9889 & 12 & $0.9944^{*}$ & 7 \\
\hline Glass & 8 & 0.6435 & 1 & 0.6394 & 6 & $0.6589^{*}$ & 6 & $0.6080^{*}$ & 1 & 0.6051 & 8 & 0.5963 & 8 \\
\hline Vowel & 10 & 0.5970 & 1 & $0.6121^{*}$ & 4 & 0.6010 & 1 & 0.6040 & 1 & 0.7030 & 4 & $0.7202^{*}$ & 4 \\
\hline
\end{tabular}

space. Thus, in the tables below, we show the average of the accuracy rates for the 10 folds for each dataset on the three LDR techniques coupled with their corresponding linear $(+\mathrm{L})$ and quadratic $(+\mathrm{Q})$ classifiers. In terms of nomenclature, the symbol $d$ indicates the dimension which yielded the highest rate, while $n$ indicates the dimension of the original data. For each classifier (linear and quadratic), the LDR method which achieved the highest average accuracy is marked with a '*'. For the comparison task a summary of the All-at-once LDR schemes presented in [8] is shown in Table 1, and the comparative details are explained subsequently.

There are considerable differences between the results for All-at-once, which is our benchmark reference, and those of Simple Voting, except for the Iris dataset (which differs only in $\mathrm{FDA}^{+} \mathrm{Q}$ from All-at-once, but with a difference which is less than 1\%). In the Pendigits dataset, we observe an improvement in all cases, specially for the linear classifier, where the average rates were approximately $10 \%$ superior when compared to those of All-at-once. As opposed to this, in the case of the quadratic classifier, the differences are not as extensive, although the improvement was higher than $1 \%$, which is good, considering the values are near $100 \%$. In the Thyroid dataset we observe that the improvement in the accuracy rates of the linear classifier on the three LDR methods was higher than $4 \%$ in 
the best case $(\mathrm{HDA}+\mathrm{L}$ and $\mathrm{CDA}+\mathrm{L})$, although the quadratic classifier did not yield an enhancement in any criteria (the difference being less than 1\%). It is interesting to note that in the case of the Wine dataset, we have the same values in both tables, where we attained the same maximum values, although these maxima were reached by different approaches. Thus, for example, in All-at-once for the linear classifier, we reached the highest value for the HDA+L $(98,89 \%)$ scheme, and in Simple Voting we attained the same maximum for the CDA ${ }^{+} \mathrm{L}$ scheme. As opposed to this, for the quadratic classifier, the highest value was obtained for the $\mathrm{HDA}^{+} \mathrm{Q}$ and $\mathrm{CDA}^{+} \mathrm{Q}(99.45 \%)$ methods, for which the corresponding value for Simple Voting was CDA+Q (99.44\%). In general, based on the results in the tables, we can state that Chernoff-based classification is the most superior.

For Weighted Voting, there are also considerable differences with the results of All-at-once, except for the Iris dataset, which differs only in FDA+Q in Allat-once, although the difference is less than 1\%. Also, in general, the results with Weighted Voting are very similar to the results of Simple Voting. In the Pendigits dataset, we observe an improvement in all cases, specially for the linear classifier average rates, which were superior by ca. $10 \%$ compared to the results of All-at-once. As opposed to this, in the case of the quadratic classifier, the differences are not as impressive, even though an improvement of more than $1 \%$ was obtained. For example, in the case of the Vowel context dataset, we have improvements in both classifiers, linear and quadratic; in the case of the linear classifier the improvements were, on the average, in all criteria by about $6 \%$; in the case of the quadratic classifier the improvements were higher than $2 \%$ in $\mathrm{HDA}^{+} \mathrm{Q}$ and $\mathrm{CDA}^{+} \mathrm{Q}$ for Weighted Voting when compared to the $\mathrm{HDA}^{+} \mathrm{Q}$ and $\mathrm{CDA}^{+} \mathrm{Q}$ entries for All-at-once. Again, similar observations about the superiority of the new Chernoff-based schemes can be observed from these tables, and are not specifically re-iterated here.

For Decision Trees, as in the previous cases, there are considerable differences with the results from All-at-once, except for the Iris dataset, which again differs only in the $\mathrm{FDA}^{+} \mathrm{Q}$ with a difference which is less than $1 \%$. In general, the results for the Decision Trees are very similar to the results of Simple and Weighted Voting. For example, in the Pendigits dataset, we observe an improvement in all cases, specially for the linear classifier average rates, which is ca. $10 \%$ more than those of All-at-once. In the case of the quadratic classifier, the differences are again not as large, although the improvement is higher than $1 \%$. An interesting behavior to observe is that the Decision Tree scheme performs as good as Voting and Weighted, while the time complexity of the former takes to classify an object is linear, against the quadratic complexity of the latter two schemes. Again, in the case of the Vowel dataset we have improvements in both classifiers, the linear and quadratic; for the linear classifier, the improvements were on the average, for all criteria, by $6 \%$, and in the case of the quadratic classifier, the improvements were less marked. In general, we infer that we can again unequivocally affirm that the Chernoff-based strategies are the most superior ones. 


\section{Conclusions}

We considered LDR techniques for the multi-class PR problem, which operate by invoking a relatively simple mapping of the problem onto a lower-dimensional subspace, leading to computationally efficient testing strategies. We have shown that it is better to solve the multi-class problem using an ensemble of Chernoffbased two-class problems, whence the overall solution is achieved by resorting to either Voting, Weighting, or a Decision Tree strategy. The experimental results obtained by testing the methods on benchmark datasets demonstrate that the Chernoff-based LDR scheme works very well for one-against-one multi-class schemes. Additionally, the proposed method is not only efficient, but also yields an accuracy comparable to that obtained by the optimal Bayes classifier.

Acknowledgments. The work of L. Rueda and C. Henríquez was supported by the Chilean National Council for Technological and Scientific Research, FONDECYT grant No. 1060904, and the work of B. J. Oommen was partially supported by NSERC, the Natural Sciences and Engineering Research Council of Canada.

\section{References}

1. Abe, S.: Support Vector Machines for Pattern Classification. Springer, Heidelberg (2005)

2. Asuncion, A., Newman, D.J.: UCI machine learning repository (2007), http://www.ics.uci.edu/ mlearn/MLRepository.html

3. Kim, S., Magnani, A., Boyd, S.: Optimal Kernel Selection in Kernel Fisher Discriminant Analysis. In: Proc. of the 23rd International Conference on Machine Learning, Pittsburgh, USA, pp. 465-472 (2006)

4. Loog, M., Duin, P.W.: Linear Dimensionality Reduction via a Heteroscedastic Extension of LDA: The Chernoff Criterion. IEEE Transactions on Pattern Analysis and Machine Intelligence 26(6), 732-739 (2004)

5. Martinez, A., Zhu, M.: Where Are Linear Feature Extraction Methods Applicable? IEEE Trans. on Pattern Analysis and Machine Intelligence 27(12), 1934-1944 (2005)

6. Rohl, M., Weihs, C.: Optimal vs. classical linear dimension reduction. In: Information Age, Studies in Classification, Data Analysis, and Knowledge Organization, pp. 252-259. Springer, Heidelberg (1999)

7. Rueda, L.: Selecting the Best Hyperplane in the Framework of Optimal Pairwise Linear Classifiers. Pattern Recognition Letters 25(2), 49-62 (2004)

8. Rueda, L., Herrera, M.: A New Approach to Multi-class Linear Dimensionality Reduction. In: Martínez-Trinidad, J.F., Carrasco Ochoa, J.A., Kittler, J. (eds.) CIARP 2006. LNCS, vol. 4225, pp. 634-643. Springer, Heidelberg (2006)

9. Rueda, L., Oommen, B.J.: On Optimal Pairwise Linear Classifiers for Normal Distributions: The Two-Dimensional Case. IEEE Transactions on Pattern Analysis and Machine Intelligence 24(2), 274-280 (2002)

10. Tax, D.M.J., Duin, R.P.W.: Using Two-Class Classifiers for Multiclass Classification. In: Proceedings of the 16th International Conference on Pattern Recognition, Quebec, Canada, vol. 2, pp. 124-127 (2002)

11. Zhu, M., Martinez, A.: Subclass Discriminant Analysis. IEEE Trans. on Pattern Analysis and Machine Intelligence 28(8), 1262-1273 (2006) 